\title{
Experimental and theoretical study of the flocculants mixing in water
}

\author{
A. Grigoryeva, Rufat S.Abiev \\ Saint Petersburg State Institute of Technology, Saint Petersburg, Russia,
}

\begin{abstract}
The article discusses the process of high molecular weight flocculant particles dissolving in water during mechanical stirring. A procedure of mixer selection taking into account mixer geometry based on the Prandtl's semi-empirical turbulence model was developed and described. It was found that the choice of the optimal rotation speed is related with the turbulent frictional stress generated by the impeller of the mixer. The results of experimental studies on the preparation of concentrated solutions of high molecular weight cationic flocculant MagnaflokM 155 using various impeller types are shown. The efficiency of a conical mixer has been confirmed, which makes it possible prepare a flocculant solution 1.3 times faster due to the possibility of mixing at higher rotational speeds with lower power consumption
\end{abstract}

\section{Introduction}

Polymers are applied in different fields of the petroleum industry to increase extraction of oil, prepare drill mud, reinforce borehole walls; they are used to enhance oil recovery to control injected water mobility. Polymer filling improves the efficiency of volumetric cleaning, reduces side channeling and losses, increases oil extraction, and reduces extracted oil cost. Coagulants and flocculants have been used for a long time during the preparation of drinking water, wastewater treatment, sludge thickening for ore-dressing plants during coal washing, and valuable mineral mining.

The major process problem during flocculation is the selection of the most efficient flocculant and the creation of optimum process conditions for flocculant solution preparation and subsequent use [1]. A lot of papers are dedicated to the investigation of different flocculant application efficiency $[2,3,4]$. At the same time, in literature and flocculant application practice there is no clear method of mixing device selection for mechanization of the solution preparation process. The imperfection of solution preparation methods results in considerable polymer ingress in suspension in the form of a gel and associates, which leads to overconsumption of expensive flocculants and reduction of their efficiency during dispersion aggregation.

Polymer action depends on the following factors:

1. Flocculant nature

2. Molecular weight

3. Dispersed phase concentration

4. Polymer adding and preparation conditions. 
Most flocculants are supplied in a solid form. It saves transportation costs and it is easier to store dry substances [5]. Perhaps, the major problem is dissolving powder flocculants that have the form of a polydisperse mixture of solid particles. As a rule, dissolving is performed in a mixing apparatus. Flocculant macromolecules are quite flexible and are present in water in the form of molecular coils that begin to uncoil, as the solution is diluted. For the coil of long polymer macromolecular chains to uncoil efficiently, good wetting of the entire surface shall be ensured.

The purpose of this paper is to devise a procedure to calculate the maximum permissible rotational speed of mixing apparatus based on theoretical and experimental studies and considering mixer geometry. At the same time, it shall be taken into account that shear stresses on the outer diameter of mixer blades shall not exceed parameters defined by flow properties of flocculants. Based on the theoretical analysis performed it is necessary to compare several types of mixing devices for the fastest dissolution.

\section{THEORETICAL PART}

The polymer dissolution rate depends on how large the particles are. It is known from the literature that polymer solution has the properties of non-Newtonian fluids [6]; however, this statement is true for high polymer concentrations. In practice, $0.1-0.3 \%$ concentrations are normally used. When analyzing the flow curves in[7], it is noted that at low concentrations gels behave almost like Newtonian fluids. Thus, in this research, we consider low-concentration flocculant solutions, whose mixing can be compared to mixing in a solid-liquid system.

For swelling and dissolution processes to proceed as fast as possible, it is necessary to keep all particles in a suspended state equally distributed throughout the entire liquid volume, at the same time, avoiding high shear forces.

In most cases, it's common practice to assess mechanical mixing intensity by the root mean square velocity gradient (shear rate)G, which is determined by formula (1) [8]:

$$
G=\left(\frac{\varepsilon}{\mu_{a}}\right)^{1 / 2},
$$

where $\varepsilon=$ power dissipated in the unit of liquid volume (specific rate of energy dissipation), $\mathrm{W} / \mathrm{m}^{3}$; and $\mu_{a}=$ dynamic viscosity of the liquid, $\mathrm{Pa} \times \mathrm{s}$.

The authors of [9] argue that the root-mean-square velocity gradient $\mathrm{G}$ shall lie within the range of 200-1000s-1, while the authors of [6]. believe that to distribute all particles throughout the volume it's enough to generate mixing, at which $G=100-400 \mathrm{~s}-1$. According to manufacturers' recommendations, there shall be two stages of mixing, when preparing a flocculant: first "fast", then "slow". However, when installing a mixer, one shall have detailed information on mixer diameter, rotational speed, the best geometry (type) of the impeller. Thus, there is no information in existing literature sources regarding the accurate selection of a mixer and mixing efficiency criteria.

According to formula (1), the root-mean-square velocity gradient depends on the power input to the apparatus. However, under turbulent conditions, the mixer's useful effect is of critical importance. Mixer geometry features were not considered in the calculation of velocity gradient based on the power dissipated in the unit of liquid volume and, therefore, velocity distribution in circumferential and axial directions was not considered either.

Mixer rotational speed increase leads to an increase in flow turbulence and particleliquid slip velocity. If flow turbulence is high enough, laminar film around the particle, in which solute concentration reaches saturation, becomes thinner, it stimulates the particle dissolution process. Nevertheless, the rotational speed increase is accompanied with an increase of shear stresses causing polymer macromolecule destruction. Therefore, mixer 
rotational speed increase above optimum value is not acceptable, since it leads to an abrupt reduction of resulting solution process properties [10]. Flocculant particle dissolution efficiency mainly depends on apparatus design and mixer rotational speed. Thus, two conflicting requirements shall be fulfilled: 1) sufficient energy for flocculant dissolution shall be supplied; 2) destruction of already uncoiled flocculant particles shall be prevented. Due to this fact, the process shall have two stages: at the beginning, mixing shall be intensive, then moderate. There is some optimum mixer rotational speed, which will be called the threshold.

The calculation procedure for mixer threshold rotational speed is based on determining Reynolds turbulent friction shear stresses generated by the mixer during operation and depending on the mixer geometry. These stresses shall not exceed shear stresses sufficient to destroy the flocculant macromolecule.

Turbulent friction stresses are calculated from semi-empirical turbulence models, the most common being the Prandtl's model, according to which turbulent friction stress $\tau_{\mathrm{T}}(\mathrm{Pa})$ is determined as

$$
\tau_{\mathrm{T}}=\rho L^{2}\left|\frac{d u}{d y}\right| \frac{d u}{d y},
$$

where $\rho=$ liquid density, $\mathrm{kg} / \mathrm{m}^{3} ; L=$ mixing length, $\mathrm{m}$; and $d u / d y=$ circumferential velocity gradient in axial direction (shear rate), $1 / \mathrm{s}$.

Substance transfer from the surface of dissolving flocculant particles to the solution volume consists of hydrodynamic and diffusion processes that are generally described by differential equations of viscous fluid dynamics and convective diffusion. The analysis of the regularity of turbulent transfer regularities in apparatuses with mixers is based on the specific features of the dynamics of mixed fluids.Considering velocity field axial symmetry and velocity profile stability in height in the main part of the volume, the coefficient of local turbulent transfer $\left(D_{\mathrm{T}}^{\pi}\right)$ at radius $\mathrm{r}$ is determined by the following formula [11]:

$$
D_{\mathrm{T}}^{Л}(r)=\alpha_{0}^{2} r_{0}^{2}\left|\frac{d u(r)}{d r}\right|,
$$

where $|(d u(r)) / d r|=$ circumferential velocity gradient on radius $r, \mathrm{~s}^{-1}$; and $\alpha_{0}=$ coefficient.

The turbulent diffusion coefficient for the peripheral mixing zone is determined by the formula (Braginsky, L.N. et al, 1984):

$$
\begin{gathered}
D_{\mathrm{T} 2}=\alpha_{0}^{2} \omega_{0} r_{0}^{2} \frac{\left(\hat{\mathrm{R}}-\hat{\mathrm{r}}_{m}\right)^{2}}{\hat{\mathrm{R}}^{2}-\hat{\mathrm{r}}_{m}^{2}}\left[1.6 \psi_{1}\left(\hat{\mathrm{r}}_{m}^{5}-1\right)+1.5 \psi_{2}\left(\hat{\mathrm{r}}_{m}^{4}-1\right)+\left(\hat{\mathrm{r}}_{m}^{2}-1\right)+\right. \\
\left.2\left(\psi_{1}+\psi_{2}+1\right) \ln \hat{\mathrm{R}}\right]
\end{gathered}
$$

where $\omega_{0}=$ angular velocity of mixer rotation, $1 / \mathrm{s} ; \hat{R}=D / d_{m}=$ main geometric criterion of similarity /ratio of apparatus diameter $D$ to mixer diameter $d_{m} ; r_{0}=$ mixer radius, $\mathrm{m} ; \hat{r}_{m}=$ $0.75 \times r_{0}=$ mean value of non-dimensional radius of division between the first and second mixing zone in the apparatus; and $\psi_{1}, \psi_{2}=$ circumferential velocity distribution parameters depending on the mixer geometry.

Equating right-hand sides of expressions (3) and (4), we obtain the formula for absolute velocity gradient:

$$
\begin{array}{r}
\left|\frac{d u}{d y}\right|=\omega_{0} \frac{\left(\hat{\mathrm{R}}-\hat{\mathrm{r}}_{m}\right)^{2}}{\hat{\mathrm{R}}^{2}-\hat{\mathrm{r}}_{m}^{2}}\left[1.6 \psi_{1}\left(\hat{\mathrm{r}}_{m}^{5}-1\right)+1.5 \psi_{2}\left(\hat{\mathrm{r}}_{m}^{4}-1\right)+\left(\hat{\mathrm{r}}_{m}^{2}-1\right)+\right. \\
\left.2\left(\psi_{1}+\psi_{2}+1\right) \ln (\hat{\mathrm{R}})\right](5)
\end{array}
$$

Mixing lengths can be calculated from the semi-empirical turbulence model proposed by Carman. According to him, $L$ can be calculated as [12] 


$$
L=k \times y,(6)
$$

where $k=0.4$ is the universal turbulence constant; and $y=\pi d m / z=$ circumferential distance between mixer blades ( $z=$ number of mixer blades).

Knowing the absolute velocity gradient and mixing length, the turbulent Reynolds stresses generated by a mixer of certain geometry at different rotational speeds can be calculated using the Prandtl formula.

Flow properties of high molecular weight flocculants were studied, and critical shear stresses for polymeric bond breakdown at different flocculant concentrations were determined in [7]. On the other hand, it is easy to plot a graph of mixer turbulent friction stress dependence at different rotational speeds for a mixer of certain geometry. The crossing point of the two graphs corresponds to defining the rotational speed of the mixer, that is, to the maximum possible rotational speed of a mixer of certain geometry, at which polymeric bonds will be preserved. Graph 1 is presented below in Fig. 1.

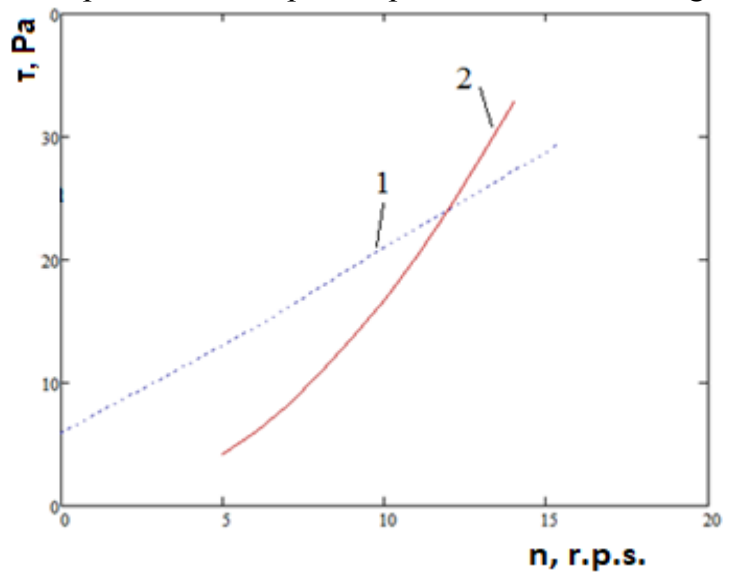

Fig. 1. Combined graph of turbulent stresses introduced by a conical mixer (line 2) and shear stresses of MagnaflockM155 flocculant (line 1) dependence on rotational speed $\mathrm{n}$.

\section{EXPERIMENTAL PART}

The concentrated solution was prepared in a cylindrical container $180 \mathrm{~mm}$ in diameter and $200 \mathrm{~mm}$ in height (at solution level of $160 \mathrm{~mm}$ ). The mixer was equipped with an electric drive of $100 \mathrm{~W}$ capacity. Distilled water of $20^{\circ} \mathrm{C}[10]$. temperature acted as a solvent.

High molecular weight cationic flocculant M 155 with a molecular weight of 8-10 mln was used as a polymer, its detailed flow characteristics are described in [7].

Dissolution of a flocculant powder in water can be considered as mixing in a liquidsolid system. For the experiment, a turbinemixer (Fig. 2a)and pitched blade mixer (Fig. $2 b)$ were chosen, since these types are recommended for solid particle mixing [11]. Also,for comparison, we chose a conical mixer(Fig. 2c), developed by us [13], since in preliminary research it demonstrated very effective operation.

Theconicalmixercontains a hub mounted on a rotating vertical shaft, on which blades with a disc are fixed; the disk is attached to the blades from below; the blades have a the shape of a curved surface with a generatrix parallel to the shaft;on the inner diameter of the blade,equipped with a curved flange for attachment to the hub, and along the outer diameter,the blades are equipped with a horizontal platform for attachment to the disc, while the disk has a central hole, and the blade has an upper edge. The blade edge is made decreasing from outer diameter of the hub to the outer diameter of the disc, and the lower 
edge of the blade is made decreasing from the outer diameter of the hub to the diameter of the hole in the disc. The angle between the tangent to blade surface atat the point of its attachment to the hub, and the tangent to the hub is in the range from 15 to $90^{\circ}$, and the angle between the tangent to the blade surface at the point with the largest diameter and the tangent to the disc surface is acute, while the blades are attached to the hub and the disc using threaded fasteners.

Standard types of mixers, i.e.,turbine mixer, pitched-blade mixer, and a conical mixer developed by us were used as impellers. All mixers were of $50 \mathrm{~mm}$ in diameter (Fig.2).

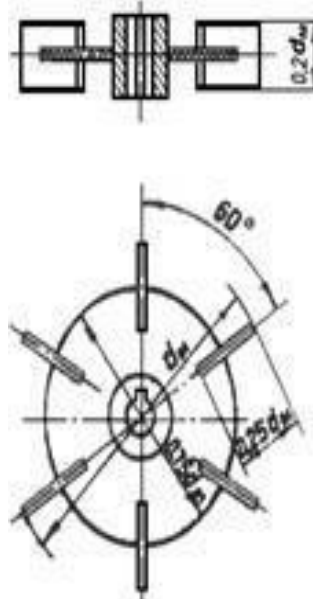

(a) (b)

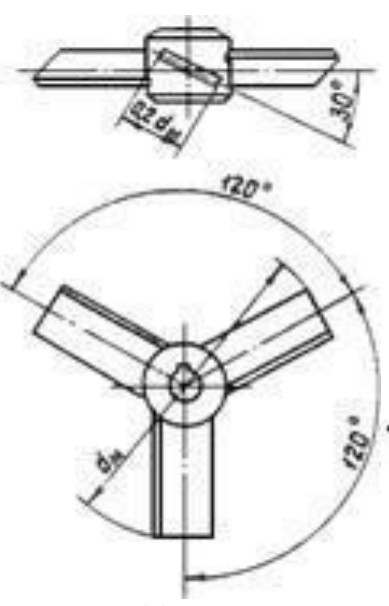

(c)
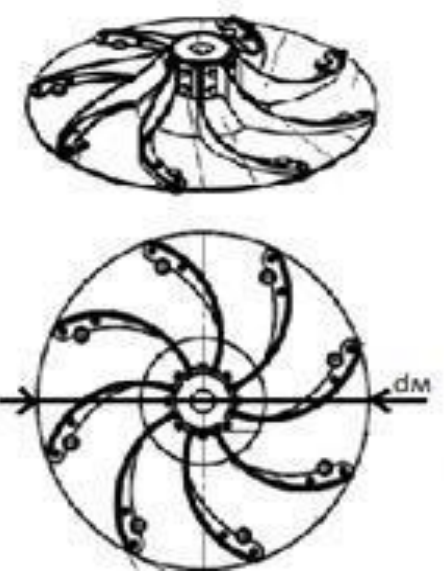

Fig. 2. Mixer types used in experiments: $\mathrm{a}$ - turbine, $\mathrm{b}$ - pitched-blade, $\mathrm{c}$ - conical.

$3 \mathrm{~g} / 1$ of powder flocculant was added to the liquid in a vessel with a pre-switched on mixer at the design speed. Thereafter, the time until complete dissolution of the polymer was recorded. $12 \mathrm{~g}$ of powder was gradually added to a 4-liter volume of liquid in the central vortex (the area in the center of the apparatus just next to the shaft) over10 seconds. In laboratory conditions, it's easy to ensure a slow and even flow of powder into the vessel with an operating mixer, directly into the central vortex. This makes it possible to wet every polymer particle with water and to prevent the formation of clumps . Adding powder to the central vortex also allows the polymer particle to be quickly distributed throughout the entire liquid volume, as particles are carried away from the central part to the periphery by centrifugal forces.

Homogeneity of the resulting solution was checked by assessment of laser beam refraction index. In homogeneous media with constant optical density, light propagation is linear. It is possible to assess the full dissolution of the flocculant by passing a directed light beam (laser) through a vessel with the solution in the process of its continuous mixing. 

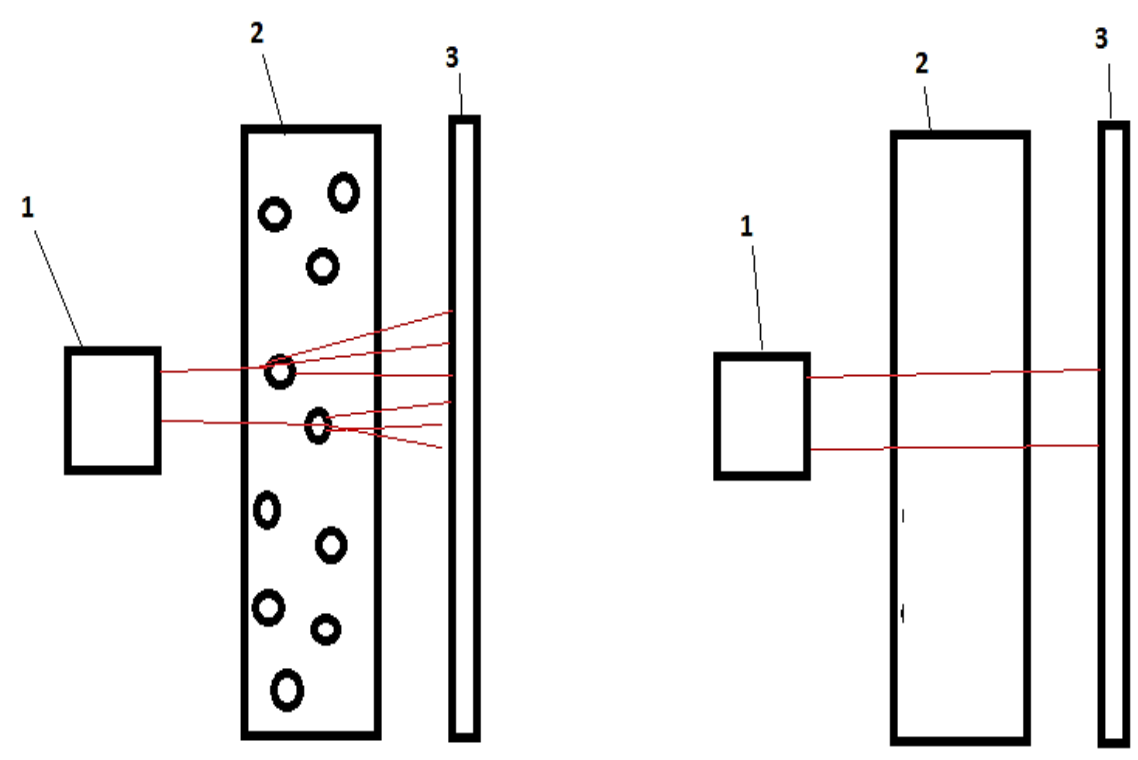

(a)

(b)

Fig. 3. Schematic layout of the unit for determination of full flocculant dissolution: a - light refraction when passing through undissolved particles, $b$ - laser light passing through the homogeneous solution.

The unit operation principle is shown in Fig. 3. If the solution is homogeneous, its optical density is the same atevery point of the volume. Thus, the laser passing through the solution from source 1 (Fig. 3) will have a constant refraction index. Visually it will be seen as a fixed laser point on shield 3 behind the vessel with the solution to be mixed 2. If the flocculant solution is heterogeneous, it contains undissolved polymeric coils impregnated with water and having a different optical density as compared to water. Since such a solution is continuously mixed, light beam refraction will vary, i.e., fluid will have a refraction index gradient. It will be seen as continuous fluctuations of laser point on the shield behind the vessel with the solution. A detailed research technique is described in [14].

To verify flocculant dissolution completion, viscosity was measured by viscometer B3246 in full compliance with GOST 9070-75. Prepared concentrated solution viscosity remained approximately the same during experiments and corresponded to data given in $[1]$.

\section{Results of studies and their discussion}

A graph of velocity gradient du/dy (in fact, it coincides with the shear rate value under the given conditions) dependence on shear stress $(\boldsymbol{\tau})$ was taken from [7]; data for $0.3 \%$ flocculant solution with a molecular weight of 5-8 mln were used. A graph of the dependence of Reynolds turbulent stresses generated by a certain geometry mixer at different rotational speeds on the shear rate was calculated by the Prandtl formula (2). Critical velocity gradient point was found on the curves crossing, above which stresses become destructive for flocculant molecules. Mixing power was calculated by the procedure described in [15]. 
Threshold rotational speed, dissolution time, and mixing power calculation results are given in Table 1.

Table 1. Results of operational efficiency calculation for different mixer types.

\begin{tabular}{llllll}
\hline Mixertype & $\begin{array}{l}\text { Threshold } \\
\text { rotation } \\
\text { speed, r.p.s. }\end{array}$ & $\begin{array}{l}\text { Power } \\
\text { for mixing- } \\
\mathrm{N}, \mathrm{W}\end{array}$ & $\begin{array}{l}\text { Apparatus } \\
\text { Dissolution } \\
\text { timeT, min } \\
\mathrm{Q},(60 / \mathrm{T})\end{array}$ & $\begin{array}{l}\text { nominal Ratio } \\
\text { capacity, Q/N, W }\end{array}$ \\
\hline \hline Conical & 12 & 0.162 & 40 & 1.5 & 7.31 \\
Three-blade & 7.5 & 0.061 & 60 & 1 & 6.32 \\
Turbine & 6 & 0.281 & 50 & 1.2 & 2.1 \\
\hline \hline
\end{tabular}

The nominal hourly capacity of apparatus $Q$ characterizes the value inverse to dissolution time T, i.e., the number of dissolution cycles per hour. Ratio $Q / N$ characterizes the number of dissolution cycles per hour for each $\mathrm{W}$ of power used for mixing. The higher is the value, the higher is the efficiency of power input into the apparatus. Therefore, the application of the conical mixer enables flocculant mixing at maximum rotational speed, as compared to other mixers studied, since it generates lower shear stresses during operation due to the streamlined shape of the blades. Application of turbine mixer at high rotational speeds is limited, since it generates dangerous shear stresses next to sharp edges of the blades, located at $90^{\circ}$ angle to the rotation plane. Apparatus with the conical mixer is not only the most efficient, but it is also the most advantageous in terms of the ratio between the useful effect (multiplicity of apparatus volumes that can be mixed during 1 hour) and expenditures (energy consumed by the mixer).

As a result of the work it was found that the use of the new conical mixer allows to speed up the process of preparing a flocculant by 1.5 times compared to the most common three-blade mixer and 1.25 times, compared to a turbine mixer. Therefore, for the proper choice of a mixer of certain geometry for the preparation or flocculant solution it is necessary to:

- $\quad$ Select mixer diameter and shaft length depending on the apparatus dimensions based on recommendations for this type of mixing apparatus for efficient suspending in the solid-liquid system

- Determine velocity gradient in the axial direction by formula (5). Velocity distribution parameters for standard mixers can be taken from reference data, for example, using RD 26-01-90-85 [16]

- Calculate mixing length by formula (6)

- Build a dependency graph for shear stresses and mixer rotational speed for the given diameter, having calculated the values by formula (2)

- Find the defining speed of mixer rotation combining the graphs. To find the defining speed, one should have data on flow properties of the flocculant solution

- Calculate the power required for mixing using the formula from RD 26-01-90-85.

It should be noted that the above procedure is acceptable only for low-concentration solutions with the properties of a Newtonian fluid.

\section{Conclusion}

A mixer selection procedure for the preparation of a concentrated polymer solution of the required concentration was developed. The theoretical calculation is based on the standard Prandtl's semi-empirical turbulence model complemented by Carman. According to calculation results using a conical mixer, flocculant can be mixed at the highest rotational 
speed compared to other impellers used in the study, since it generates lower shear stresses during operation due to the streamlined shape of the blades. Application of a turbine mixer at high rotational speeds is limited, as it generates dangerous shear stresses due to the sharp edges of the blades located at $90^{\circ}$ to the rotation plane. The apparatus with a conical mixer is also the most advantageous in terms of the ratio of the useful effect and the consumed energy.

\section{References}

1. L. V. Gandurina Wastewater treatment using synthetic flocculants. Monograph. 198 p. (M.: CJSC DAR / VODGEO, 2007)

2. S. Mohd, A. Hamidi, F. Mohd Floc behavior and removal mechanisms of cross-linked. Duriozibethinus seed starch as a natural flocculant for landfill leachate coagulationflocculation treatment. Waste Management, 74, 362-372 (2018)

3. W. Zhen, H. Wenxiu, Y. Guihua Preparation of cellulose-based amphoteric flocculant and its application in the treatment of wastewater. Carbohydrate Polymers, 215(1), 179-188 (2019)

4. T. Zhenle, Z. Liping, S. Xinxin, Sh. Gang, Ni. Caihua Preparation and flocculation performance study of a novel amphoteric alginate flocculant. Journal of Physics and Chemistry of Solids, 141, 109-118 (2020)

5. V. S. Frolov, V. I. Udovitsky Preparation of powdered flocculants at coal processing enterprises of Kuzbass. Mountain Information and Analytical Bulletin, 7, 240-243 (2009)

6. Yu. I. Weizer, D. M. Mints High molecular weight flocculants in wastewater treatment. 2nd ed. reslave. and add, 200 p. (M.: Stroyizdat, 1984)

7. E. V. Ulrich, T. V. Shevchenko, A. N. Pirogov, Yu. S. Midutsina The study of the rheological properties of flocculants. The chemicallindustry today, 5, 25-28 (2011)

8. M. Hentse Wastewater treatment: Transl. from English by T.P. Mosolova, 480 p. (M.: Mir, 2006)

9. A. K. Zapolsky, A. A. Baran Coagulants and flocculants in water purification processes: Properties. Receiving. Application. 208 p. (L.: Chemistry, 1987)

10. V. I. Aksenova, Yu. V. Anikin The use of flocculants in water systems: a training manual, 98 p. (Yekaterinburg: USTU-UPI, 2008)

11. L. N. Braginsky, V. I. Begachev, V. M. Barabash Mixing in liquid media, 336 p. (L.: Chemistry, 1984)

12. B. Shi-i Turbulent flow of liquids and gases. Per. from English M.G. Morozova, 345 p. (M.: Publishing house of foreign literature, 1962)

13. R. Sh., Abieb, A. N. Grigoryeva Mixing device. RF Patent,no. 2683078, (2019)

14. G. L. Evmenova, A. A. Baychenko Homogenization of solutions of high molecular weight polymers during flocculation of coal dispersions. Report at the Miner Week 98 symposium, 128-129 (M.: MGGU, 1998)

15. A. N. Grigoryeva, R. Sh. Abiev The influence of the geometry of the mixing device on the diameter of air bubbles during mixing in the gas-liquid system. The Chemical Industry Today, 5, 18-22 (2020)

16. RD 26-01-90-85 "Mechanical mixing devices. Calculation Method." 